\title{
Current magnification effect in mesoscopic systems at equilibrium
}

\author{
Colin Benjamin and A. M. Jayannavari \\ Institute of Physics, Sachivalaya Marg, Bhubaneswar 751 005, Orissa, India
}

(Dated: October 31, 2018)

\begin{abstract}
We study the current magnification effect and associated circulating currents in mesoscopic systems at equilibrium. Earlier studies have revealed that in the presence of transport current (nonequilibrium situation), circulating currents can flow in a ring even in the absence of magnetic field. This was attributed to current magnification which is quantum mechanical in origin. We have shown that the same effect can be obtained in equilibrium systems, however, in the presence of magnetic flux. For this we have considered an one-dimensional open mesoscopic ring connected to a bubble, and the system is in contact with a single reservoir. We have considered a special case where bubble does not enclose magnetic flux, yet circulating currents can flow in it due to current magnification.
\end{abstract}

PACS numbers: 73.23.Ra, 5.60.Gg, 72.10.Bg

Mesoscopic physics deals with the realm which is in between the microscopic (atomic or molecular) scale and macroscopic one. In these systems quantum phase coherence length $L_{\phi}$ exceeds the sample size $L$. These systems have provided several, often counter-intuitive new results exploring truly quantum effects beyond the atomic realm 1 2. These systems are expected to reveal the crossover between quantum and the macroscopic classical regimes, which is of fundamental interest. The notion of intrinsic decoherence and dephasing of a particle interacting with its environment are beinforactively pursued and experimentally being analysed 3 . The decoherence mechanism signals the limit beyond which the system dynamics approaches the classical behavior. One of the prominent mesoscopic effect is that of observation of persistent currents in metallic rings enclosing magnetic flux. Büttiker, Imry and Landauer predicted 1 the existence of equilibrium persistent current in an ideal onedimensional metallic ring in presence of magnetic flux, with a period of $\phi_{0}, \phi_{0}$ being the elementary flux quanta $h c / e$. The existence of persistent currents have been verified experimentally . Persistent surpentegcur in both

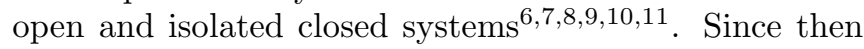
circulating currents have been predicted in open systems in presence of a transport current. This phenomenon is associated with current magnification effect in mesoscopic rings 10.11.12. For this we consider a metallic loop connected to two reservoirs by two ideal leads. Transport current $I$ flows through the system when the two reservoirs are kept at different chemical potentials, say $\mu_{1}$ and $\mu_{2}$ respectively. The upper and lower arms of the ring are of different lengths and currents $I_{1}$ and $I_{2}$ flow in these such that $I_{1} \neq I_{2}$. The basic law of current conservation namely, Kirchoff's law demands that $I=I_{1}+I_{2}$. In the classical case both $I_{1}$ and $I_{2}$ are positive and flow along the direction of the applied chemical potential. However, when quantum mechanically currents are calculated depending upon the length parameters it is found that for particular values of Fermi energy $I_{1}$ (or $I_{2}$ ) can be much larger than $I$. Current conservation thus dictates $I_{2}$ (or $I_{1}$ ) to be negative such that $I=I_{1}+I_{2}$. The property that current in one of the arms is larger than the transport current is referred to as current magnification effect. This quantum effect has no classical analog in equilibrium. In such a situation one can interpret that the negative current flowing in one arm continues to flow as a circulating current in the loop 10. 11.12 . Our procedure of assigning a circulating current is exactly the same as the procedure well known in classical LCR ac network analysis. When a parallel resonant circuit(capacitance $\mathrm{C}$ connected in parallel with a combination of inductance $\mathrm{L}$ and resistance $\mathrm{R}$ ) is driven by external electromotive force(generator), circulating currents arise in the circuit at resonant frequency 13 . The magnitude of the negative current in one of the arms flowing against the direction of the applied current is taken to be that of the circulating current. When the negative current flows in the upper arm the circulating current direction is taken to be anticlockwise (or negative) and when it flows in the lower arm the circulatimemrent direction is taken to be clockwise(or positive) 10.11 .22 .13$.

It should be noted that these circulating currents arise in the absence of magnetic flux and in presence of transport currents (i.e., in a non-equilibrium system). It has also been shown that impurities affect current magnification in a non-trivial way. In fact, impurities can enhance current magnification as opposed to the conventional wisdom that impurities would degrade current magnification 10.12. Studies on circulating currents in mesoscopic open rings have been extended to thermal currents 14 and to-spin currents in the presence of Aharonov-Casher flux 1 . Recently this effect has been studied in presence of spin-flip sfattering which causes dephasing of electronic motion 1210.

In the present work we are interested in the basic question, whether current magnification can occur in equilibrium systems. For this we consider the system as depicted in Figure (1). The static localised flux piercing the loop is necessary to break the time reversal symmetry and induce a persistent current in the system. The geometry we consider is a one-dimensional ring coupled to a bubble. The system is connected to a reservoir at chemical potential $\mu$. The reservoir acts as an inelastic scatterer and as a source of energy dissipationt. We would like 


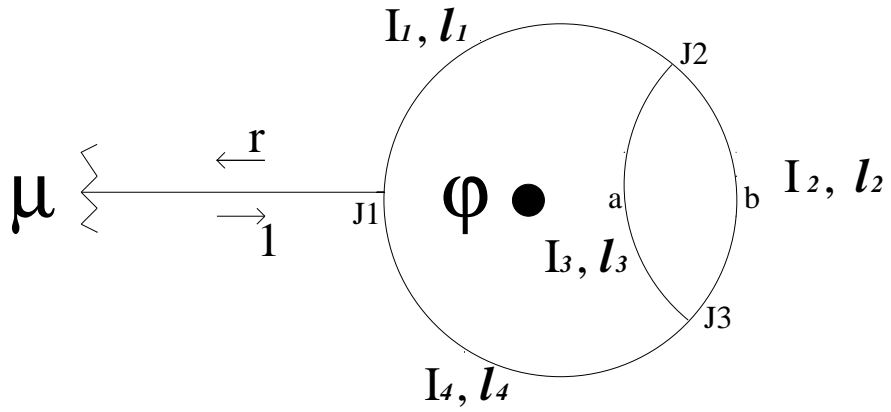

FIG. 1: One dimensional mesoscopic ring coupled to a bubble with a lead connected to a reservoir at chemical potential $\mu$. The localised flux $\phi$ penetrates the ring.

to emphasize that the magnetic flux is localised in a finite region. The loops J1J2aJ3J1 and J1J2bJ3J1 enclose the localised flux $\phi$. However, the bubble J2aJ3bJ2 does not enclose the flux $\phi$. The special situation we have considered, is to answer the question of existence of circulating currents in equilibrium systems. We show that circulating currents(due to current magnification) arise in a bubble which does not enclose a magnetic flux. We would like to mention here that the current magnification effect and the associated circulating currents arise even when the magnetic field extends over the entire sample. However, for this the treatment is involved as one has to study separately persistent as well as circulating currents in the bubble as they have different symmetry properties. This has been studied in a simple loop in the presence of both transport currents and magnetic flux 11 .

In the local coordinate system the wavefunctions in the various regions of the ring in absence of magnetic flux are given as follows

$$
\begin{aligned}
& \psi_{0}=e^{i k x_{0}}+r e^{-i k x_{0}}, \\
& \psi_{1}=a e^{i k x_{1}}+b e^{-i k x_{1}}, \\
& \psi_{2}=c e^{i k x_{2}}+d e^{-i k x_{2}}, \\
& \psi_{3}=e e^{i k x_{3}}+f e^{-i k x_{3}}, \\
& \psi_{4}=g e^{i k x_{4}}+h e^{-i k x_{4}} .
\end{aligned}
$$

Here $x_{i}, i=0, . .4$ are coordinates along the connecting lead to the reservoir, and the segments J1J2, J2bJ3, J2aJ3, and J3J1 respectively. The Fermi wavevector is defined as $k=\sqrt{2 m E / \hbar^{2}}$. To solve for the unknown coefficients in eqn.(1) we use Griffith 17 boundary condition at the junctions $J 1, J 2$ and $J 3$. These boundary conditions are due to the single-valuedness of wavefunction and current conservation (Kirchoff's law). In the presence of magnetic flux in the system we can choose a gauge for the vector potential in which the field does not appear explicitly in the Hamiltonian. The boundary conditions do not change, however the electron propagating from one junction to another picks up an additional phase, which is positive for clockwise motion and negative for anti-clockwise motion, but of same magnitude. For further details see Refs. 10,18]. Naturally different segments pick up different phases. Using the above mentioned boundary conditions we get

$$
\begin{array}{r}
1+r=a+b e^{-i \alpha_{1}}=g e^{i k l_{4}+i \alpha_{4}}+h e^{-i k l_{4}}, \\
1-r-a+b e^{-i \alpha_{1}}+g e^{i k l_{4}+i \alpha_{4}}-h e^{-i k l_{4}}=0, \\
a e^{i k l_{1}+i \alpha_{1}}+b e^{i k l_{1}}=c+d e^{i \alpha_{2}}=e+f e^{i \alpha 3}, \\
a e^{i k l_{1}+i \alpha_{1}}-b e^{-i k l_{1}}-c+d e^{-i \alpha_{2}}-e+f e^{-i \alpha_{3}}=0 \\
c e^{i k l_{2}+i \alpha_{2}}+d e^{-i k l_{2}}=e e^{i k l_{3}+i \alpha_{3}}+f e^{-i k l_{3}}=g+h e^{-i \alpha_{4}}, \\
c e^{i k l_{2}+i \alpha_{2}}-d e^{-i k l_{2}}+e e^{i k l_{3}+i \alpha_{3}}-f e^{-i k l_{3}}-g+h e^{-i \alpha_{4}}=0 .(2
\end{array}
$$

Here $\alpha_{1}, \alpha_{2}, \alpha_{3}$ and $\alpha_{4}$ are phases picked up by the wavefunctions in the segments J1J2, J2bJ3, J2aJ3 and J3J1 respectively and we have $\alpha_{1}+\alpha_{2}+\alpha_{4}=2 \pi \phi / \phi_{0}$, and $\alpha_{1}+\alpha_{3}+\alpha_{4}=2 \pi \phi / \phi_{0}$, such that $\alpha_{2}=\alpha_{3}$ as required by definition. Using eqn.(2) we have solved for all the unknown coefficients in eqn.(1).

In the lead connecting the reservoir to our circuit there is no current flow as $|r|^{2}=1$. Throughout the discussion the lengths are scaled with respect to the total length of the bubble $l=l_{2}+l_{3}$. The wavevector $k$ is identified in a dimensionless form $k \equiv k l$. The probability current density is defined as $J=\frac{e \hbar}{2 m i}\left(\psi^{*} \nabla \psi-\psi \nabla \psi^{*}\right)$. For the circuit segment $J 1 J 2$ of the figure (1), when we derive the probability current density we get- $J=\frac{e \hbar k}{m}\left(|a|^{2}-\right.$ $\left.|b|^{2}\right)$. Now the current densities $(I)$ in their dimensionless form are given by dividing $J$ by $\frac{e \hbar k}{m}$. This approach is widely used in literature to define the current densities, see Refs. [7] 11]. The current densities in the small interval $d k$ around the Fermi energy $k$ in the various segments of the circuit are given by -

$$
\begin{aligned}
& I_{1}=|a|^{2}-|b|^{2}, \\
& I_{2}=|c|^{2}-|d|^{2}, \\
& I_{3}=|e|^{2}-|f|^{2}, \\
& I_{4}=|g|^{2}-|h|^{2} .
\end{aligned}
$$

Just to mention again that $I_{1}, I_{2}, I_{3}$ and $I_{4}$ are the persistent current densities in the segments $J 1 J 2, J 2 b J 3, J 2 a J 3$ and $J 3 J 1$ respectively. The persistent current densities in various parts of the circuit show cyclic variation with flux and $\phi_{0}$ periodicity, and oscillate between positive and negative values as a function of energy or the wavevector $k$ as expected. Since the analytical expressions for these currents are too lengthy we confine ourselves to a graphical interpretation of the results. It should be noted that in all these currents flux enters only through the combinations $\alpha_{1}+\alpha_{2}+\alpha_{4}$ and $\alpha_{1}+\alpha_{3}+\alpha_{4}$ the magnitude of these combinations is given by $2 \pi \phi / \phi_{0}$ as expected. For us the current densities in the bubble(J2bJ3aJ2) are of special importance as in this region there is a possibility of current magnification which will be analysed below. The currents induced in segment J3J1 and J1J2 are equal, i.e $I_{1}=I_{4}$. These currents may have positive(clockwise) or negative(anticlockwise) values depending on the flux $\phi$ and value of Fermi wavevector $k$. For a fixed $k$ this current oscillates between positive 


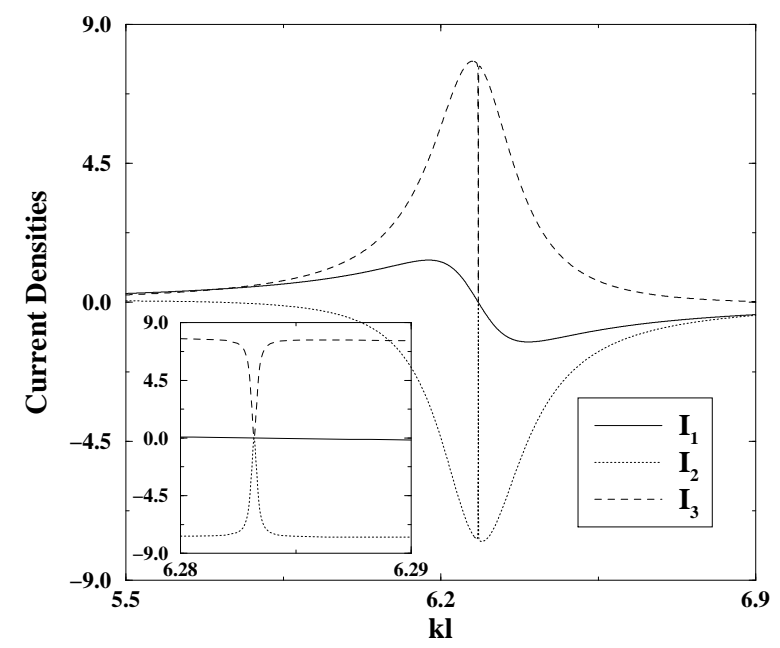

FIG. 2: Persistent current densities are shown as function of $k l$. The lengths are $l_{1} / l=l_{4} / l=0.25, l_{2} / l=0.45, l_{3} / l=0.55$ and flux $\phi=0.1$. In the inset we have shown the current densities around the value wherein $I_{1}$ goes to zero.

and negative values as a function of $\phi$ with a period $\phi_{0}$ and are asymmetric in $\phi$. Similarly for fixed value of $\phi$ currents oscillate as one varies $k$. The magnitude of current shows a maximum or minimum near the corresponding eigen-states of the system. We have calculated these eigen states for two different cases. For open system as depicted in figure (1) one can calculate the energies(or wavevector) of these states by looking at the poles of the S-Matrix. These states correspond directly to resonances. In our case S-Matrix is simply a complex reflection amplitude $r$. We have also analysed the eigen states of a closed system(without coupling lead to reservoir) by wavefunction matching in various segments using waveguide theory. The eigenvalues are obtained by solving the following equation, resulting from waveguide theory,

$$
\begin{aligned}
\cos (\alpha)= & \frac{1}{\cos \left(k l_{-}\right)}\left(\cos k\left(l_{1}+l_{+}\right)-\right. \\
& \left.\frac{1}{4} \frac{\sin \left(k l_{1}\right) \sin \left(k l_{2}\right) \sin \left(k l_{3}\right)}{\sin \left(k l_{+}\right)}\right),
\end{aligned}
$$

where, $\alpha=2 \pi \phi / \phi_{0}, l_{+}=\left(l_{2}+l_{3}\right) / 2$ and $l_{-}=\left(l_{2}-\right.$ $\left.l_{3}\right) / 2$.

We analyse the case of a bubble with unequal lengths, of its two arms, i.e., the length of $J 2 b J 3 \neq J 2 a J 3$. This asymmetry implies that current densities in the two arms of the bubble $I_{2} \neq I_{3}$. In figure (2), we plot the persistent current densities in various parts of the circuit. It should be noted that absolute value of the persistent current densities $I_{2}$ and $I_{3}$ are individually much larger than the input current density $I_{1}$ into the bubble and thus the current magnification effect is evident(without violating the basic Kirchoff's law). The input current arises due to the presence of flux $\phi$ as it breaks the time

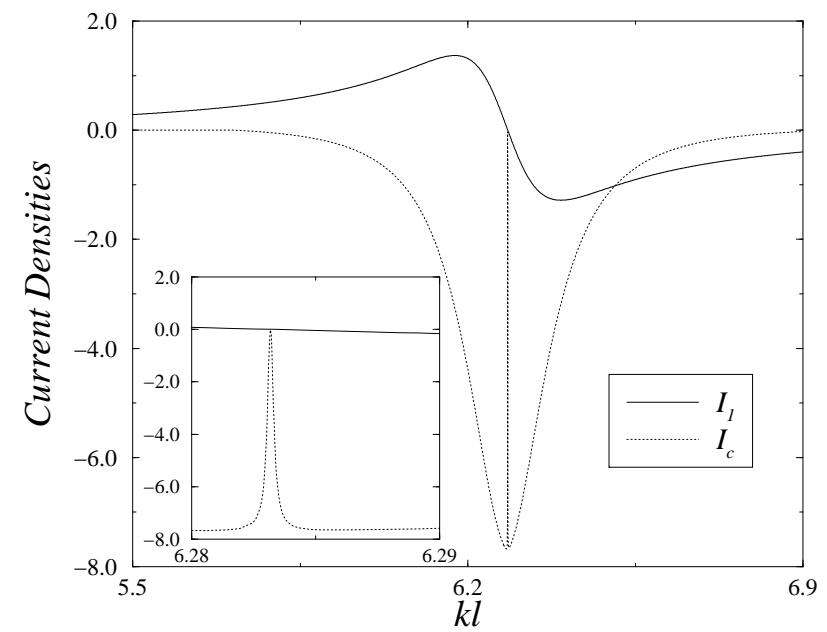

FIG. 3: Persistent current density $I_{1}$ and circulating current density $I_{c}$ is plotted as function of $k l$. The parameters are same as used in fig. 2. The inset shows the behavior of $I_{c}$ and $I_{1}$ around their zero values.

reversal symmetry. The physical parameters used for this figure are mentioned in the figure caption. In the interval $5.5<k l<6.9$ the current $I_{1}$ changes from positive to negative and exhibits extremum around the real part of the poles of the S-Matrix (6.278 and 6.328). For the closed system the eigen values are at 5.93 and 6.68 . The difference between eigenvalues for closed and open systems(quasi bound states) arise from the additional scattering from the junction $J 1$ coupled to the reservoir. Moreover, eigenvalues for open systems are complex, as electron has a finite lifetime in the ring system before entering into the reservoir. When $I_{1}$ is positive, negative current density of magnitude $I_{2}$ flows in the arm $J 2 b J 3$ of the bubble. Thus, when $I_{1}$ is positive circulating current flows in the anti-clockwise direction in the bubble. In the range where $I_{1}$ is negative, i.e, input current into the bubble is in anticlockwise direction, then positive current flows in arm $J 2 a J 3$. According, to our convention as mentioned earlier, circulating current flows in the anticlockwise direction. The magnitude of this circulating current density $I_{c}$, is taken to be the value of current in one of the arms of the bubble moving against the input current into the bubble as explained in detail in the introduction.

In figure (3) we have plotted the persistent current density $I_{1}=I_{4}$ and the circulating current density $I_{c}$ in the bubble for the same parameters used in figure 2. It should be noted that if we interchange the values of $l_{2}$ and $l_{3}$ keeping other parameters unchanged circulating current will flow in a clockwise direction. This is obvious from the geometry of the problem.

We generally observe current magnification at those Fermi energy wavevector intervals around the eigen energies of the system 10.11 . However, there are some exceptions. In figure (4), we plot one of those exceptions. 


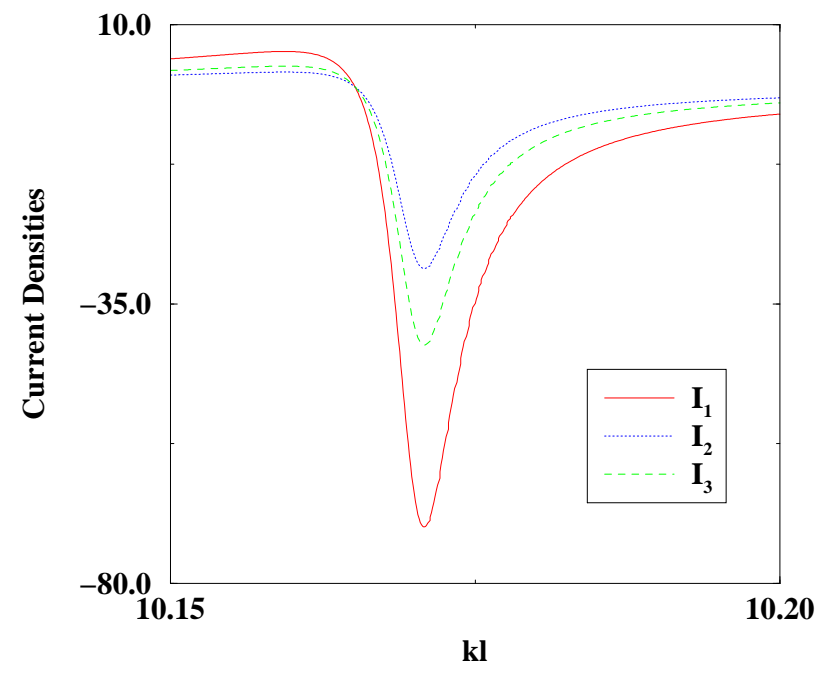

FIG. 4: Persistent current densities are platted as function of $k l$. The lengths are $l_{1} / l=l_{4} / l=0.25, l_{2} / l=0.15, l_{3} / l=0.85$. Flux $\phi=0.1$.

The new physical parameters are mentioned in the figure caption. In figure(4) we show that current magnification does not occur at places which are eigen values of the aforesaid system. Here the real part of the eigen wavevector $k l$ corresponds to 10.184 (for closed system it is at 10.171). One can readily notice that the magnitude of persistent current (i.e, input current $I_{1}$ ) shows extrema around this value. Around this region both the currents in the bubble $I_{2}$ and $I_{3}$ are individually smaller than $I_{1}$ and they flow in the same direction as the input current. Hence we do not observe current magnification effect around this quasi bound state of the open system. We also observe that current magnification does occur at some places which are not near the eigen values of the system.

All these figures establish the fact that the current magnification effect (and associated circulating currents) which are quantum mechanical in origin are extremely sensitive to the system parameters. The exact conditions for current magnification cannot be readily predicted a priori. The orbital magnetic moment of the system is given by the line integral of the total current taken across the entire system. The total current is given by integrating the current densities upto the Fermi energy (at temperature $T=0$ ). If the system exhibits current magnification effect one should be able to detect it experimentally by observing the enhanced response of the magnetic moment by appropriate tuning of Fermi energies. We expect systems comprising several metallic loops interwoven together to exhibit a new feature in the magnetic response due to current magnification. It should be noted if the whole system is embedded in a magnetic field then we have both persistent currents as well as circulating currents that can be separated by their symmetry properties under flux reversal 11 . Just for the sake of simplicity and to show the existence of current magnification in equilibrium we have taken a system in which bubble does not enclose a magnetic flux, which may not be an ideal system. However, it clarifies our contention.

In conclusion we have shown that current magnification effect can occur in equilibrium mesoscopic systems in presence of magnetic flux. Earlier, it was shown to occur in a non-equilibrium state10. This quantum effect is extremely sensitive to system parameters. Our system also exhibits breakdown of parity effects (using eqn. (4) 6. This, along with analysis of current magnification in presence of magnetic flux, encompassing the entire sample will be reported elsewhere.
* Electronic address: colin@iopb.res.in

$\dagger$ Electronic address: jayan@iopb.res.in

1 Y. Imry, Introduction to Mesoscopic Physics (Oxford University, New York, 1997).

2 P. S. Deo and A. M. Jayannavar, Pramana J. Phys.56, 439 (2001).

3 D. Sprinzak, et.al, Phys. Rev. Lett. 84, 5820 (2000).

4 M.Büttiker, Y.Imry and R.Landauer, Phys. Lett. A 96, 365 (1983);

${ }^{5}$ L. P. Levy, et.al, Phys. Rev. Lett. 64, 2074 (1990).

${ }^{6}$ H. F. Cheung and E. K. Riedel, Phys. Rev. B40, 9498 (1989).

7 M. Büttiker, Phys. Rev. B32, 1846 (1985).

8 T. P. Pareek and A. M. Jayannavar, Phys. Rev. B54, 6376(1996).

9 P. S. Deo and A. M. Jayannavar, Mod. Phys. Lett. B7, 1045 (1993).
10 A. M. Jayannavar and P. S. Deo, Phys. Rev. B49, 13685 (1994);T. P. Pareek, P. S. Deo and A. M. Jayannavar, Phys. Rev. B52, 14657 (1995).

11 A. M. Jayannavar, P. S. Deo, and T. P. Pareek, Physica B212, 216 (1995).

12 C. Benjamin, et.al., Mod. Phys. Lett. B15 ,19 (2001).

13 D. F. Shaw, An Introduction to Electronics (Longman, 2nd ed., London, 1970) p. 51.

14 M. V. Moskalets, Euro. Phys. Lett. 41, 189 (1998).

15 T. Choi, C. M. Ryu and A. M. Javannavar, Int. J. Mod. Phys. B12, 2091 (1998) and cond-mat/9808245.

16 S. K. Joshi, D. Sahoo and A. M. Jayannavar,Phys. Rev. $\mathbf{B}(2001)$ in press.

17 J-B. Xia, Phys. Rev. B45, 3593 (1992).

18 Y. Gefen, Y. Imry, and M. Ya. Azbel, Phys. Rev. Lett. 52, 129 (1984). 